\title{
Future of translational research: Why go pragmatic?
}

\author{
This article was published in the following Dove Press journal: \\ Pragmatic and Observational Research \\ 7 February 20II \\ Number of times this article has been viewed
}

\author{
Abdul Rahman Jazieh \\ King Saud Bin Abdulaziz University \\ for Health Sciences, Riyadh, Kingdom \\ of Saudi Arabia
}

\begin{abstract}
Translational research has succeeded in expanding our medical knowledge and bringing many discoveries to the clinic. However, the prevailing research enterprise is plagued with many challenges that may limit the ability to realize the full potential benefits of these advances. The main challenge of current research approaches is the inability to reach all potential candidates who may benefit from the findings in a timely and systematic way. There are many factors contributing to this limitation, including the differences between the study population and the research setting from larger, more heterogeneous populations in variable health care settings. Understanding the current research limitations may help in developing more practical research interventions that are relevant to real-life patients in real-life settings. The adoption of pragmatic research culture will include designing new and innovative approaches for research projects, maximizing the benefits of the existing research methodologies, and enhancing the research infrastructure. The gap between the discovery and the intended patients should be bridged by pragmatic research approaches that bring a timely and appropriate benefit to those who need it the most. This paper presents the limitations of the current research enterprise and the suggested solutions to adopt more pragmatic approaches.
\end{abstract}

Keywords: translational research, methodology, pragmatic research

\section{Introduction}

The advances in health sciences have been monumental, especially over the last couple of decades. These advances are the outcome of numerous high quality research efforts. So-called "translational research" has succeeded in bringing many discoveries from the bench to the clinic and, to a lesser extent, vice versa. But the question that arises is: Did we optimize our research system to the extent that we can keep doing what we are doing? It has been successful so far, so why change?

While experts may take different sides in a debate about the pros and cons of the current research process, one point that everyone would agree on is that there is the need to improve the research process at various levels. This is even more pressing to optimize the wide use and application of research at the practice level to reach the intended target population. The purpose of this paper is not to provide a suggestion for a quick fix for the insurmountable research challenges, but rather focus on one area that is not well addressed in the classic medical research activities, namely "pragmatic research".

Pragmatic research can be defined in different ways, but it simply means conducting research in real-life settings so one can get real-life results. It is the methodology of research that aims at identifying the right and relevant question and pursues the 
answer through approaches that assure obtaining adequate knowledge on the applicability and generalizability of the conclusion to the intended population. In order to highlight the importance of adopting pragmatic research, two issues will be addressed in this manuscript, ie, why pragmatic research is needed and how to do it?

\section{Why pragmatic research is needed}

The answer to this question comes from the fact that most of the current research approaches do not precisely reflect the true life situation of the whole population of intended patients, especially during drug development. The following are a few examples illustrating this point:

- Only a small fraction of patients is enrolled in clinical trials. For example, 2\%-3\% of cancer patients participate in clinical studies in the US.$^{1,2}$ Furthermore, certain population may even be underrepresented in these studies. ${ }^{3-5}$

- Clinical trials may have restricted inclusion criteria that are suitable for only a small subgroup of a patient population, yet the approval and real-life use will invariably include different patient populations. ${ }^{6}$

- The registration trials are generally conducted in advanced tertiary centers with many resources, support staff, and services which may not exist to the average practitioner, who may not have access to expensive technologies, sophisticated expertise, or a supportive infrastructure while they are managing their patients in day-to-day practice.

- The research staff helps in managing the patients enrolled in clinical trials and the protocol provides guidance to the treating physicians. This explains why patients in clinical trials may fare better than those who are not in studies. ${ }^{7-9}$ Research staff will not be available to help practitioners when they are treating patients off clinical trials.

- Conducting a study is a rigorous process, including auditing and monitoring, and many issues that may be overlooked in real-life practice, such as drug accountability, drug usage, and drug-drug or drug-food interaction risk.

- Postmarketing pharmacovigilance programs/studies are not adequate because they depend on the abilities and willingness of physicians to recognize the safety issues and voluntarily report them. That is why, in many instances, safety issues are recognized only after patients suffer serious or even fatal side effects.

- Ethnicity and pharmacogenomic variability pose another layer of complexity, because therapeutic studies may be conducted in certain populations (such as Western or Asian); yet the studied drug gets approved and used by people from different races and ethnicities across the globe.

- Access to innovative therapies that are complex and expensive may be limited in certain areas or for certain populations, which makes these discoveries or advances irrelevant to some people who may be deprived of lifesaving therapies.

These facts highlight the need to incorporate more pragmatic research approaches at various steps of drug development and other clinical research activities. This will be critical for the evolution of translational research to achieve the best possible outcome for patients.

\section{What is the solution?}

Pragmatic research will help address the real-life performance and activity of new therapeutic modalities. Therefore, the current model of translational research should be modified to accommodate more pragmatic research concepts. This can be achieved by a number of actions. Certainly thinking and acting outside the traditional box will open new horizons that have the potential to transform the research efforts into more effective endeavors. The following are examples of new emerging approaches that have the potential to do just that.

\section{Developing and adopting innovative research approaches}

- Creating and adopting innovative concept design is critical to identify the most appropriate patient population that will benefit potentially from the medications. ${ }^{10,11}$ Creating such studies will require a new approach to study design from hypothesis generation to data analysis. Developing more pragmatic clinical trials that focus on the most relevant outcome, including more real-life patients, is important to achieve more generalizable results. ${ }^{12}$ Furthermore, pragmatic research studies should be conducted as early as possible in the regulatory process of drug approval in order to obtain real-life data from different settings and real patient populations. This will assure collecting data about the efficacy and safety of a particular therapy in a systematic way rather than in a haphazard sketchy way.

- Facilitating access to curative therapies may require developing a therapeutic approach using available resources, such as less expensive medication or shorter duration of therapy. This approach may save many lives or alleviate the suffering of many people rather than waiting for more expensive therapies to become available. For example, children in developing countries are dying from malignancies due to lack of access to expensive 
treatment. A protocol using a less expensive, readily available medication may prove to be effective in saving the lives of children afflicted with this disease. ${ }^{13-16}$ Another example is an investigation to decrease the duration of radiotherapy for early breast cancers which will not only be more feasible for patients in countries with limited resources but also more convenient for patients in developed countries. ${ }^{17,18}$

- The design and analysis of these studies require proper and accurate statistical methodology that aims at reaching meaningful results with value in real life not just focusing on statistical significance of differences between numbers.

\section{Revamping the infrastructure of research}

It is critical to involve the largest possible samples of the target population from different backgrounds. This will entail encouraging multiple site trials at national levels and across borders. Therefore, cooperative research group support is required. Developing practice-based research through practice-based research networks may help to identify the gap between recommended care and actual care, and become like a laboratory to test new discoveries in the real world. Furthermore, having electronic systems that are linked and able to communicate in a proper way to generate databases will enable conducting real-life-real-time research on specific issues. Enhancing information technology to improve research is paramount to enable us to move to large-scale, broad-based, and real-life research. ${ }^{19-22}$

\section{Improving current research approaches}

The aim is to build on the existing experiences and knowledge by utilization of classic methodology and approaches on a larger scale and in a more effective way to paint a better picture of the performance of a certain therapy or condition in the real world. The following are examples of such approaches.

- Improve the utilization of the "expanded-access programs" which provide medications to patients prior to approval for marketing. However, expanded access programs usually follow the strict inclusion criteria submitted to the regulatory agencies. In a sense, this may have certain limitations regarding who would be included in the drug evaluation, but nevertheless, it is closer to real life by mere access to a larger patient population in different settings, ${ }^{23}$ therefore, a better utilization of this process by collecting detailed data and conducting assessments and analyses to delineate different issues related to the wider use of new therapies.

- Encourage the use of retrospective studies which may help evaluate the efficacy and safety of therapies already used in practice, generate new hypotheses, and/or determine the outcomes of certain diseases. However, this approach has many inherent limitations, eg, the inability to capture data in a systematic and complete way and to identify all reasons for drug interruption, dose modification, and adverse events. In spite of all these limitations, retrospective data or even case reports may provide new insights about certain medical conditions or therapies that are noteworthy. Properly conducted retrospective studies will help tapping and mining already existing data that might reveal useful information in an easy-to-do, ie, a more feasible way, than other study types.

- Another approach is to conduct prospective observational studies on a large group of patients whom physicians decided to treat with a certain therapy, then monitor efficacy and safety closely without intervening in the management decision. Analysis of the findings will reveal any concern related to treatment safety or efficacy, in addition to determining the "real-life" patterns of practice.

- Accelerate the validation of therapy or intervention in different patient populations prior to wide use, especially in different ethnic groups. Due to differences in pharmacogenomics among ethnic groups, retesting medications is prudent to determine safety and efficacy.

\section{Conclusion}

Adopting research approaches that bring about a meaningful benefit to the intended patient population in real-life setting is long overdue. This includes expanding and improving the currently used approaches, in addition to developing new and innovative methods that bring the most benefit to those who need it the most. It is about bringing the right intervention to the right patient at the right time, and that is the essence of "pragmatic research".

\section{Disclosure}

The author reports no conflict of interest in this work.

\section{References}

1. Craig BM, Gilbert SM, Herndon JB, et al. Participation of older patients with prostate cancer in Medicare eligible trials. J Urol. 2010;184(3): 901-906.

2. Murthy VH, Krumholz HM, Gross CP. Participation in cancer clinical trials: Race-, sex-, and age-based disparities. JAMA. 2004;291(22): 2760-2766.

3. Hutchins LF, Unger JM, Crowley JJ, et al. Underrepresentation of patients 65 years of age or older in cancer-treatment trials. $N$ Engl J Med. 1999;341(27):2061-2067. 
4. Tejada HA, Green SB, Trimble EL, et al. Representation of African-Americans, Hispanics, and whites in National Cancer Institute cancer treatment trials. J Natl Cancer Inst. 1996;88(12):812-816.

5. Christian MC, Trimble EL. Increasing participation of physicians and patients from underrepresented racial and ethnic groups in National Cancer Institute-sponsored clinical trials. Cancer Epidemiol Biomarkers Prev. 2003; $12: 277 \mathrm{~s}-283 \mathrm{~s}$

6. Fern LA, Whelan JS. Recruitment of adolescents and young adults to cancer clinical trials - international comparisons, barriers and complications. Semin Oncol. 2010;37(2):e1-e8.

7. Halbreich U, Smail N, Tu X. Participation in clinical trials may improve care of acute schizophrenia inpatients in a general hospital. CNS Spectr. 2008;13(9):757-761.

8. Janni W, Kiechle M, Sommer H. Study participation improves treatment strategies and individual patient care in participating centers. Anticancer Res. 2006;26(5B):3661-3667.

9. McKenna RJ Jr. The impact of clinical trial protocols on patient care in a community hospital. Cancer. 1993;72(9 Suppl):2828-2833.

10. Chabner B. New results will change the paradigm for phase I trials and drug approval. Oncologist. 2010;15(10):1023-1025.

11. Hamburg M, Collins F. The path to personalized medicine. $N$ Engl J Med. 2010;22:363(4):301-304.

12. Mullins C, Montgomery R, Tunis S. Uncertainty in assessing value of oncology treatments. Oncologist. 2010;15 Suppl 1:58-64.

13. Stefan DC, Stones D. How much does it cost to treat children with Hodgkin lymphoma in Africa? Leuk Lymphoma. 2009;50(2): 196-199.

14. Hunger SP, Sung L, Howard SC. Treatment strategies and regimens of graduated intensity for childhood acute lymphoblastic leukemia in low-income countries: A proposal. Pediatr Blood Cancer. 2009;52(5):559-565.
15. Kazembe P, Hesseling PB, Griffin BE, et al. Long term survival of children with Burkitt's lymphoma in Malawi after cyclophosphamide monotherapy. Med Pediatr Oncol. 2003;40(1):23-25.

16. Hesseling PB. The SIOP burkitt lymphoma pilot study in Malawi, Africa. Med Pediatr Oncol. 2000;34(2):142.

17. Whelan TJ, Pignol JP, Levine MN, et al. Long-term results of hypofractionated radiation therapy for breast cancer. $N$ Engl J Med. 2010; 362(6):513-520.

18. Bentzen SM, Agrawal RK, Aird EG, et al; START Triallists' Group. The UK standardization of breast radiotherapy (START) Trial B of radiotherapy hypofractionation for treatment of early breast cancer: A randomized trial. Lancet. 2008;371(9618):1098-1107.

19. Rosati K. Using electronic health information or pharmacovigilance: the promise and the pitfalls. J Health Life Sci Law. 2009;2(4): 171-173.

20. Schneeweiss SS. A basic study design for expedited safety signal evaluation based on electronic healthcare data. Pharmacoepidemiol Drug Saf. 2010;19(8):858-868.

21. Maro JC, Platt R, Holmes JH, et al. Design of a national distributed health data network. Ann Intern Med. 2009;151(5):341-344.

22. Westfall JM, Mold J, Fagnan L. Practice-based research-"Blue Highways" on the NIH Roadmap. JAMA. 2007;297(4):403-406.

23. Chahal M. Off-trial access to experimental cancer agents for the terminally ill: Balancing the needs of individuals and society. $J$ Med Ethics. 2010:36(6):367-370.
Pragmatic and Observational Research

\section{Publish your work in this journal}

Pragmatic and Observational Research is an international, peer-reviewed, open access journal that publishes data from studies designed to reflect more closely medical interventions in real-world clinical practice compared with classical randomized controlled trials (RCTs). The manuscript management system is completely online and includes a very quick and fair peer-review

\section{Dovepress}

system. Visit http://www.dovepress.com/testimonials.php to read real quotes from published authors. 